\title{
Simulation of Lower Hybrid Current Drive for DEMO
}

\author{
Ali Asghar Molavi Choobini1 ${ }^{*}$, Ahmad Naghidokht ${ }^{2}$, Zahra Karami ${ }^{3}$ \\ ${ }^{1}$ Shahrekord Branch, Sama Technical and Vocational Training College, Islamic Azad University, \\ Shahrekord, Iran \\ ${ }^{2}$ Department of Physics, Isfahan University of Technology, Isfahan, Iran \\ ${ }^{3}$ Zanjan Branch, Department of Engineering, Islamic Azad University, Zanjan, Iran \\ Email: ${ }^{*}$ aliasghar.molavi64@gmail.com
}

Received 27 July 2014; revised 26 August 2014; accepted 8 September 2014

Copyright (C) 2014 by authors and Scientific Research Publishing Inc.

This work is licensed under the Creative Commons Attribution International License (CC BY).

http://creativecommons.org/licenses/by/4.0/

(c) () Open Access

\section{Abstract}

Lower hybrid (LH) wave is not only convenient to generate a flat or reversed magnetic shear profiles, but also helps one to explore scenarios for steady-state tokamak operation with improved confinement. Here with LSC code (lower hybrid simulation code), we calculate density and temperature profiles, relative power of injected wave and current wave lunch for two options of DEMO at the launched LH wave frequency $5 \mathrm{GHz}$. Two plasma scenarios pertaining to two different DEMO options, known as pulsed (option 1) and steady-state (option 2) models, have been analyzed. We perceive that power deposition by using lower hybrid wave injection mainly takes place near the edge of plasma and approximately in more peripheral region for both of options but has approximately higher efficiency for option 1 compared to option 2 . About current wave lunch, a major part of that is close to the plasma edge for both of options. We have some considerable parts that reach to internal layers for option 1 and then current drive mainly takes place in a wider, more peripheral region for option 1.

\section{Keywords}

Lower Hybrid Waves, Tokamak, Current Drive, Fokker-Planck Equation

\section{Introduction}

Steady-state operation of a fusion power plant requires external current drive to minimize the power requirements and a high fraction of bootstrap current is required. One of these external sources for current drive is low-

*Corresponding author. 
er hybrid current drive (LHCD) that has been widely applied in many tokamaks. Among the three well-proven radio-frequency (rf) heating and current drive (H\&CD) systems using electromagnetic wave, i.e., ion cyclotron resonance frequency (ICRF), electron cyclotron resonance frequency (ECRF), and lower hybrid (LH) current drive (LHCD), the latter exhibits the highest experimental current drive efficiency [1] [2]. It is well known that the mechanism of LHCD to drive the current is related to the electrons of very large energy, so called the fast electrons. In the LHCD theory, when the resonance condition $\omega-k_{\|} v=0$ is fulfilled, the LHW will generate fast electrons which carry large parallel moment and form the current through parallel electron landau damping. The LHCD often results in strongly non-Maxwell electron distributions. A major concern about the use of LHCD as a tool for thermonuclear fusion research on tokamaks exists in the observed failure of coupled LH power when operating at reactor-grade high plasma densities due to LHCD cannot penetrate high density plasmas because klystron sources near technology limit. This problem was solved by a method assessed on Frascati tokamak upgrade [3]. Recent experiments on lower hybrid ( $\mathrm{LH}$ ) penetration at reactor relevant densities together with the recent demonstrations of the technological viability of the passive-active multi junction launcher on long pulses, have removed major concerns about the employment of LH waves on next generation tokamaks [4], where LH could profitably drive far-off axis plasma currents, allowing current profile control and helping in sustaining burning performance. The launcher technology concept "passive-active multi junction" whose effectiveness was demonstrated for the first time on FTU at $8 \mathrm{GHz}$ [5] has been recently validated on long pulses on Tore Supra at 3.7 GHz [6], thus dissipating the latest doubts about its employment during steady-state operations in reactor-relevant machines. With high performance plasmas in large-size tokamaks like ITER, where LH deposition takes place in the outer half of the plasma [7], a means of driving off-axis current is essential to achieve advanced regimes [2].

In this paper with the aim of being prepared for the design phase of the next experimental reactors, investigations of using LH on DEMO have been done. The role of DEMO in the "fast track development of fusion" is roadmap to fusion power, gap analysis of development needs in the physics and technology understanding and capabilities and fusion development issues. DEMO is the last "research machine" before a commercial fusion power plant. DEMO must show fusion is economically and environmentally acceptable. In this work we focus on the so-called option 1 and option 2 of DEMO [8] to consider the assessment of the LHCD for them. Option 1 is the DEMO pulsed model, where a transformer drives the main current, and option 2 is related to "optimistic" DEMO design pointing at steady-state operations that are at the upper limit of achievable ITER performance. Option 2, compared to its conservative counterpart (option 1), entails the most demanding challenges that the fusion community may expect in LHCD systems during the next years.

\section{Simulation and Results}

In this paper lower hybrid current drive physics have been assessed with the help of LSC code, allowing us to calculate the sensitivity of LH power deposition and current-driven profiles on several parameters. The LSC suit is based on a set of mutually coupled codes consisting of a ray-tracing tool and a quasi-linear Fokker-Planck code [9]. LSC is a computational model for lower hybrid wave current drive based on FORTRAN programming language in which, electrons and ions heating, geometric details and plasma profile are discussed and space effects of two-dimensional phase of the wave spectrum injected in Fokker-Planck equation is approximated in order to simulate the desired parameters. LSC code that we have used here is approximated to one dimension (parallel to magnetic field) and for accessing better results, perpendicular temperature must be considered too. We have related parameters of two options of DEMO in Table 1 [8].

Here we choose injection of $5 \mathrm{GHz}$ waves. The choice of LHCD frequency results from a delicate trade-off between manifold counteracting elements: several physics issues demand to move the frequency as high as possible, while technological limitations put some upper bounds. Some mechanisms also entail deleterious effects for LHCD [10]. Referring to the simulations performed for different ITER scenarios shows that accordingly, albeit no calculation have been carried out for DEMO yet, a LHCD system for this machine can be hardly conceived with a frequency lower than $5 \mathrm{GHz}$ [4]. Also given a certain power to be launched through a port, multipactor also constrains the operational frequency. Coming to technological issues, $5 \mathrm{GHz}$ currently represent the highest frequency that suitable, reliable, high power RF sources (i.e. klystrons) are expected to achieve in a reasonable time. Alternatively the $4.6 \mathrm{GHz}, 250 \mathrm{~kW}$ klystrons developed represent a back-up solution [4]. Although $5 \mathrm{GHz}$ sources are not fully developed and the location of DEMO together with its alternating current 
Table 1. Basic parameters for two options of DEMO.

\begin{tabular}{ccc}
\hline & Tokamak & \\
\hline Parameters & DEMO 1 & DEMO 2 \\
\hline $\mathrm{R}(\mathrm{cm})$ & 960 & 850 \\
$\mathrm{a}(\mathrm{cm})$ & 240 & 283 \\
B, toroidal field (T) & 7.45 & 5.84 \\
$I_{p}(\mathrm{MA})$ & 14.4 & 19.1 \\
$T_{e 0}(\mathrm{keV})$ & 57 & 53 \\
$n_{e 0}\left(\mathrm{~cm}^{-3}\right)$ & $16.8 \times 10^{13}$ & $15 \times 10^{13}$ \\
\hline
\end{tabular}

distribution grid is not known, a solution close to the one proposed for ITER can be reasonably envisaged. We have LH frequencies that suitable for specific tokamaks like FTU (8 GHz), Alcator C-mode (4.6 GHz), EAST (2.45 GHz), JET (3.7 GHz), Tore Supra (3.7 GHz), KSTAR (5 GHz) and $5 \mathrm{GHz}$ that proposed in ITER (accordingly for DEMO) [4].

\subsection{Fokker-Planck Equation}

With injection LH wave and increasing energy of plasma particles, Coulomb collisions of plasma particles increase with each other. The effect of such collisions is described with adding a quasi-linear term to Vlasov equation, which is called Fokker-Planck equation and give a general description of the distribution function changes due to successive collisions. When the correlation between the fluctuating fields is small, the evolution of a distribution of charged particles $f(\vec{r}, \vec{p}, t)$ can be described by an electron kinetic equation can be written as:

$$
\frac{\partial f_{e}}{\partial t}=\left(\frac{\partial f_{e}}{\partial t}\right)_{c}+\left(\frac{\partial f_{e}}{\partial t}\right)_{w}
$$

That the ()$_{c}$ term is the Coulomb collision and the ()$_{w}$ term is the wave diffusion (quasi-linear) operator. In first step we plotted normalized density and temperature profiles (density normalized to $n_{e 0}$ and temperature to $T_{e 0}$ ) for option 1 of DEMO [8] versus normalized distance from the plasma center in Figure 1 . We can see the peaked behavior of these profiles and reducing of them in the edge of plasma as like as we expect.

\subsection{Relative Power of the Injected Wave Spectrum}

Since the coupling of lower hybrid waves with plasma particles depends on various factors such as distance from the edge of plasma and the parallel diffraction index, we calculated relative power of normalized injected wave versus above factors for two options of DEMO, which we introduced in previous section, in Figure 2 and Figure 3. In Figure 2 and Figure 3 we calculated the power deposition profile from the edge to the plasma center. As we see in this figure, the injected wave spectrum can penetrate into the plasma from the edge. Owing to the peaked behavior of the density profile, the accessibility condition allows the waves to reach a more internal layer. This is also due to the lower plasma temperature which causes the waves to be absorbed on main peak around $x / R \cong 0.25$ for option 1 and with lower efficiency around $x / R \cong 0.28$ for option 2 which are close to the edge of plasma. However, deposition takes place in a wider, more peripheral region for both of options.

\subsection{Current Wave Lunch}

Electron current drive at any flux surface corresponds to [9]:

$$
J_{r f}=\frac{-e n_{e}}{\Gamma} \int \mathrm{d} v_{\|} D_{q l}\left(v_{\|}\right) \frac{\partial f_{e}\left(v_{\|}\right)}{\partial v_{\|}} \frac{4 v_{\|}^{3}}{5+z}\left[\mu-\frac{1+z / 2+3 \mu^{2} / 2}{3+z} \frac{v_{\|}^{2}}{v_{r}^{2}}\right]
$$

where quasi-linear coefficient is $D_{q l}\left(v_{\|}\right)=\sum_{s} \frac{\pi}{2}\left(\frac{e}{m_{e}}\right)^{2} E_{\|}^{2} \delta\left(\omega-k_{\|} v_{\|}\right)\left(E_{\|}\right.$is the wave field amplitude parallel 

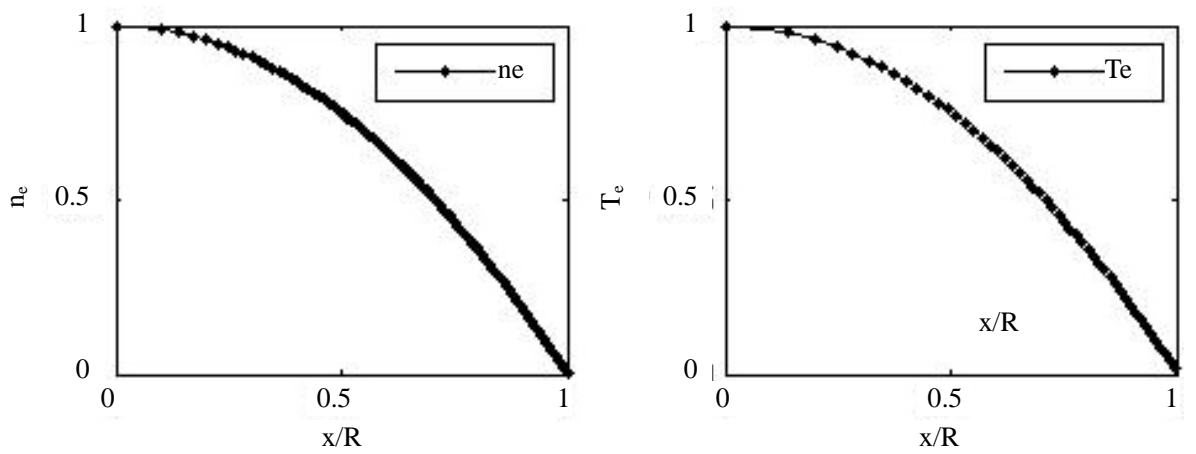

Figure 1. Normalized density (ne) and temperature (Te) profiles for option 1 of DEMO versus normalized distance from the plasma center.

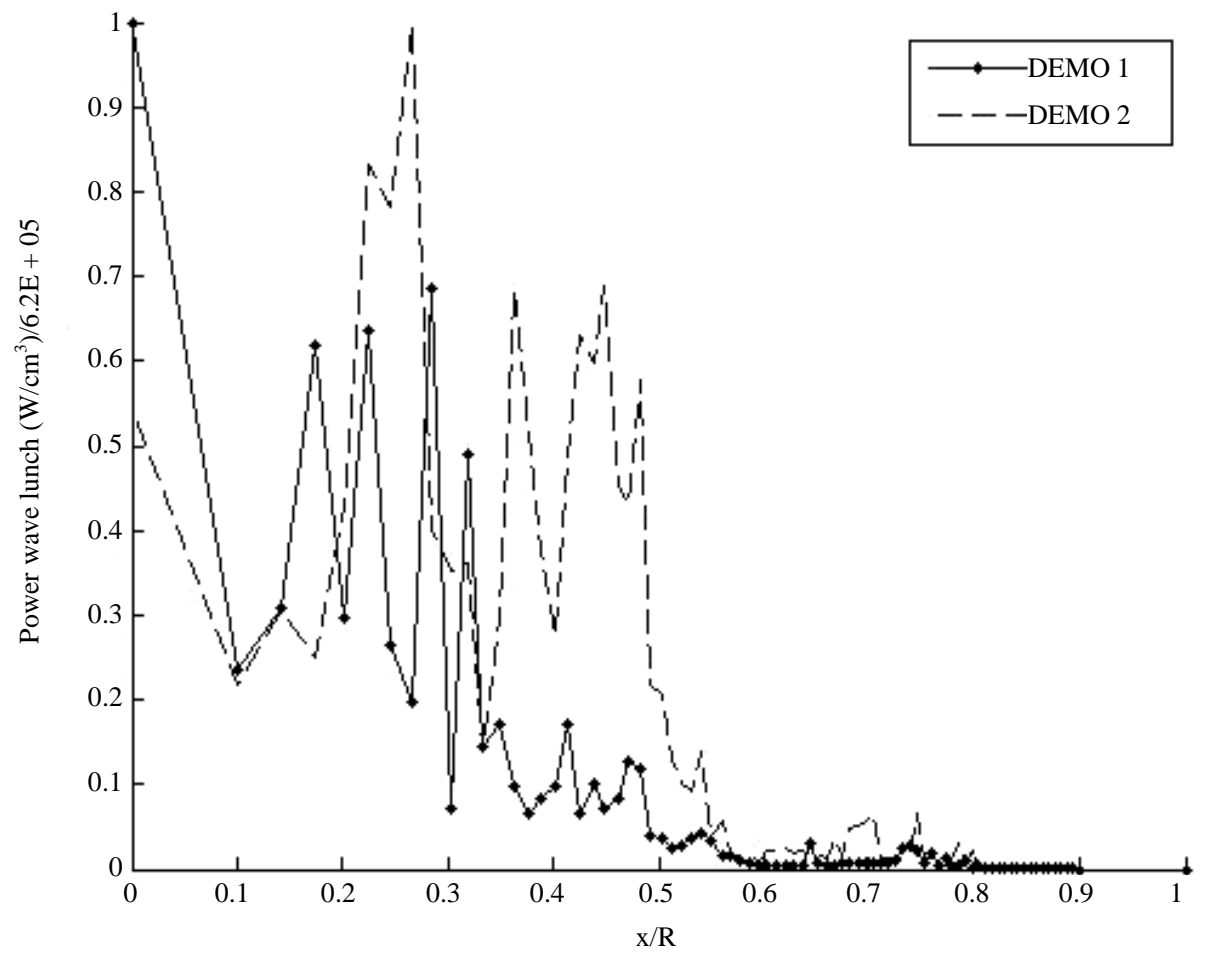

Figure 2. Normalized power wave lunch for two options of DEMO at the launched LH wave frequency $5 \mathrm{GHz}$ versus distance from the edge of plasma.

to the magnetic field, $k_{\|}$is parallel wave number), $f_{e}\left(v_{\|}\right)$is electron distribution function. $\mu=+1$ and $\mu=-1$ identify anti-parallel direction of current drive to the field, respectively. With investigating in this equation, we perceive that current drive depends on various parameters such as effective charge and particle velocity (and then distance from the edge of plasma) that have different values in plasma and therefore we will have different currents. We plotted the current wave lunch at a relative distance from the plasma center in versus normalized distance $(x / R)$ for two options of DEMO such that a distance was measured from the exterior part of tokamak and the location of the wave injection. As seen in Figure 4 current drive have a positive peak which indicates a maximum resonance mode and shows that optimal conditions for the coupling of waves with plasma particles. This coupling by Landau damping mechanism causes the loss of wave energy and transfers wave momentum to plasma particles and plasma heating. A major part of current drive is close to the plasma edge, near $x / R \cong 0.1$ for option 2 and $x / R \cong 0.25$ for option 1 . We have some considerable parts that reach to internal layers at $x / R \cong 0.38,0.43,0.49$ for option 1 and then current drive mainly takes place in a wider, more peripheral region for option 1 . 


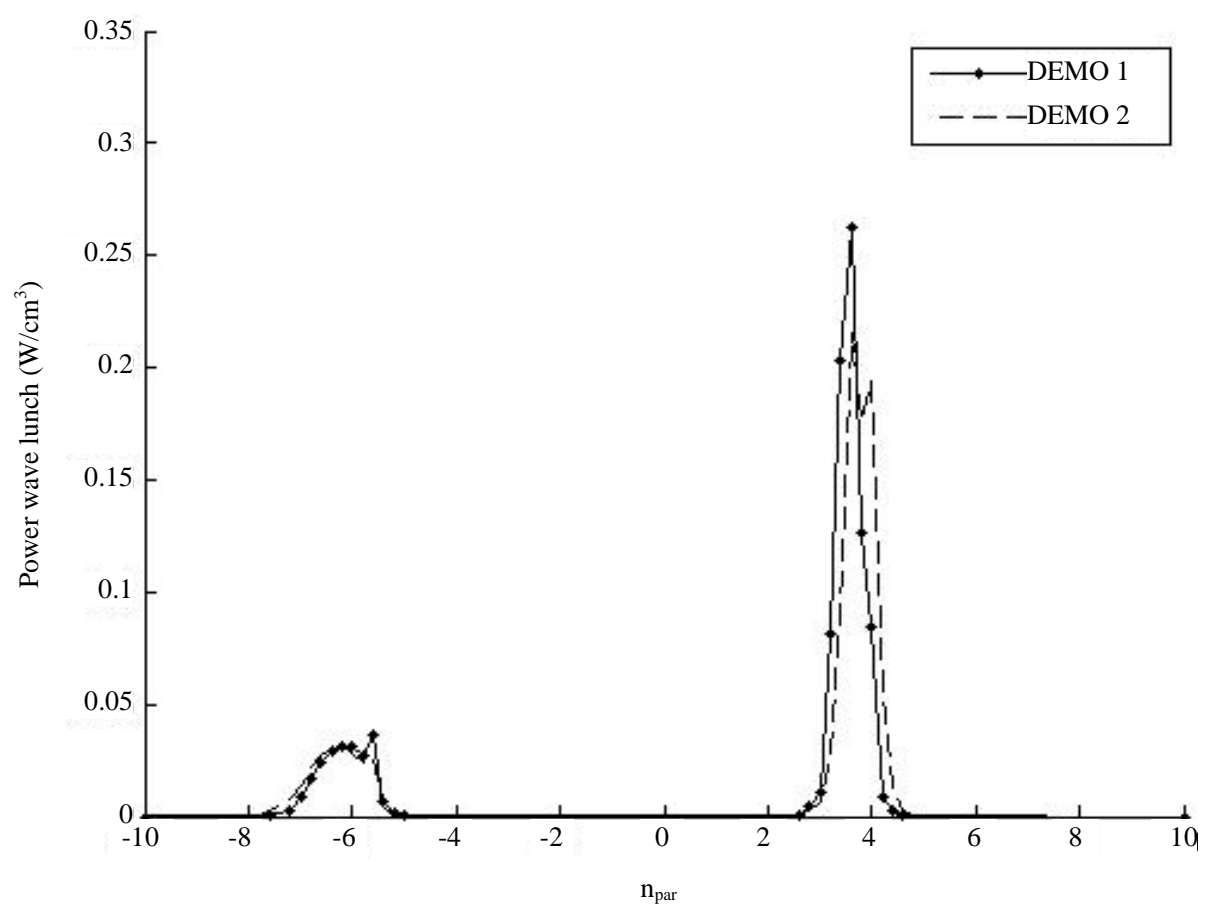

Figure 3. Normalized power wave lunch for two options of DEMO at the launched LH wave frequency $5 \mathrm{GHz}$ versus parallel diffraction index.

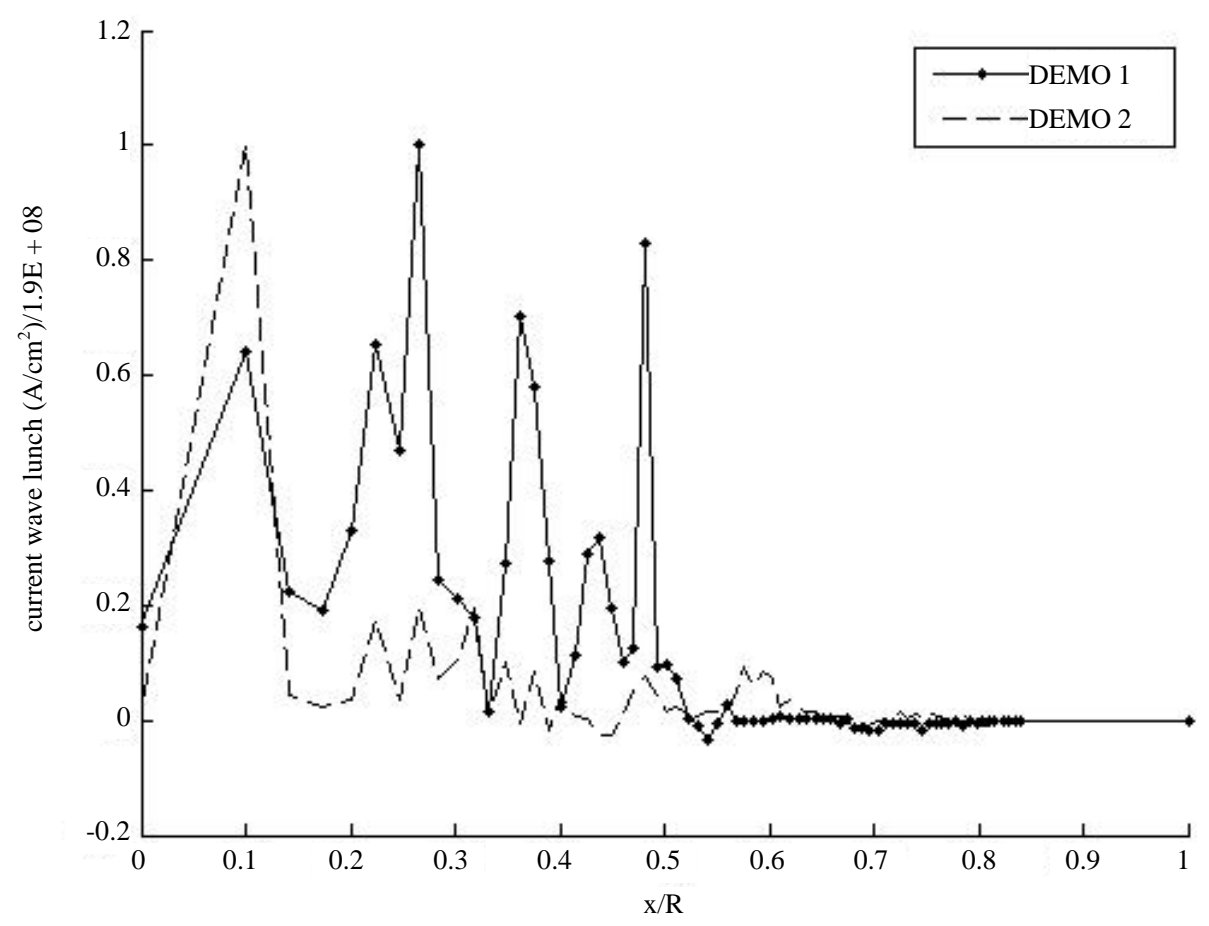

Figure 4. Normalized current wave lunch for two options of DEMO at the launched LH wave frequency $5 \mathrm{GHz}$ versus distance from the edge of plasma.

\section{Discussion}

Because of its advantage of simplicity and efficiency, a lower-hybrid-current drive (LHCD) has been widely used in tokamak experiments. Lower hybrid current drive (LHCD) has proven to be one of the most efficient 
ways to generate non-inductive current in tokamak experiments. Two plasma scenarios pertaining to two different DEMO options, known as pulsed (option 1) and steady-state (option 2) models, have been analyzed. According to results for relative power of injected wave, wave absorption has major peaks near the edge of plasma for both of options but with higher efficiency for option 1 although we have access to more internal layers for option 2. However, and with considering of parallel diffraction index spectrum for injected wave, deposition takes place in a wider, more peripheral region for both of options. About current drive, major part of that is close to the plasma edge for both of options. We have some considerable parts that reach to internal layers for option 1 and then current drive mainly takes place in a wider, more peripheral region for option 1 . Our results are approximate since LSC code that we have used here is approximated to one dimension (parallel to magnetic field) and for accessing better results, perpendicular temperature must be considered too. However, these results give us good insight and are in fairly good agreement with results for ITER [7].

\section{Acknowledgements}

This work is supported by Sama technical and vocational training college, Islamic Azad University, Shahrekord Branch and Isfahan University of Technology.

\section{References}

[1] Decker, J., Peysson, Y., Hillairet, J., Artaud, J.F., Basiuk, V., et al. (2011) Calculation of Lower Hybrid Current Drive in ITER. Nuclear Fusion, 51, Article ID: 073025. http://dx.doi.org/10.1088/0029-5515/51/7/073025

[2] Gormezano, C., Sips, A.C.C., Luce, T.C., Ide, S., Becoulet, A., Litaudon, X., et al. (2007) Steady State Operation. Nuclear Fusion, 47, S285. http://dx.doi.org/10.1088/0029-5515/47/6/S06

[3] Cesario, R., amicucci, L., Cardinali, A., Castaldo, C., Marinucci, M., et al.; the FTU Team (2010) Current Drive at Plasma Densities Required for Thermonuclear Reactors. Nature Communications, 1, 274.

[4] Ceccuzzi, S., Barbato, E., Cardinal, A., et al. (2013) Lower Hybrid Current Drive for DEMO. Physics Assessment and Technology Maturity. Fusion Science and Technology, 64, 748.

[5] Pericoli, R.V., Bibet, PH., Mirizzi, F., Apicella, M.L., Barbato, E., Buratti, P., Calabro, G., et al. (2005) LHCD and Coupling Experiments with an ITER-Like PAM Launcher on the FTU Tokamak. Nuclear Fusion, 45, 1085. http://dx.doi.org/10.1088/0029-5515/45/9/008

[6] Ekrdhal, A., delpech, L., Goniche, M., Guilhem, D., Hillairet, J., Preynas, M., et al. (2010) Validation of the ITERRelevant Passive-Active-Multijunction LHCD Launcher on Long Pulses in Tore Supra. Nuclear Fusion, 50 Article ID: 112002. http://dx.doi.org/10.1088/0029-5515/50/11/112002

[7] Hoang, G.T., Becoulet, A., Jacquinot, J., Artaud, J.F., Bae, Y.S., Beaumont, B., et al. (2009) A Lower Hybrid Current Drive System for ITER. Nuclear Fusion, 49, Article ID: 075001. http://dx.doi.org/10.1088/0029-5515/49/7/075001

[8] Poli, E., Tardind, G., Zohm, H., Fable, E., Farina, D., Figini, L., Marushchenko, N.B. and Porte, L. (2013) Electron Cyclotron Current Drive Efficiency in DEMO Plasmas. Nuclear Fusion, 53, Article ID: 013011. http://dx.doi.org/10.1088/0029-5515/53/1/013011

[9] Ignat, D.W. and Redd, A.J. (2000) Lower Hybrid Simulation Code Manual. Plasma Physics Laboratory, Princeton University, Princeton.

[10] Barbato, E. and Santini, F. (1991) Quasi-Linear Absorption of Lower Hybrid Waves by Fusion-Generated Alpha Particles. Nuclear Fusion, 31, 673. http://dx.doi.org/10.1088/0029-5515/31/4/005 
Scientific Research Publishing (SCIRP) is one of the largest Open Access journal publishers. It is currently publishing more than 200 open access, online, peer-reviewed journals covering a wide range of academic disciplines. SCIRP serves the worldwide academic communities and contributes to the progress and application of science with its publication.

Other selected journals from SCIRP are listed as below. Submit your manuscript to us via either submit@scirp.org or Online Submission Portal.
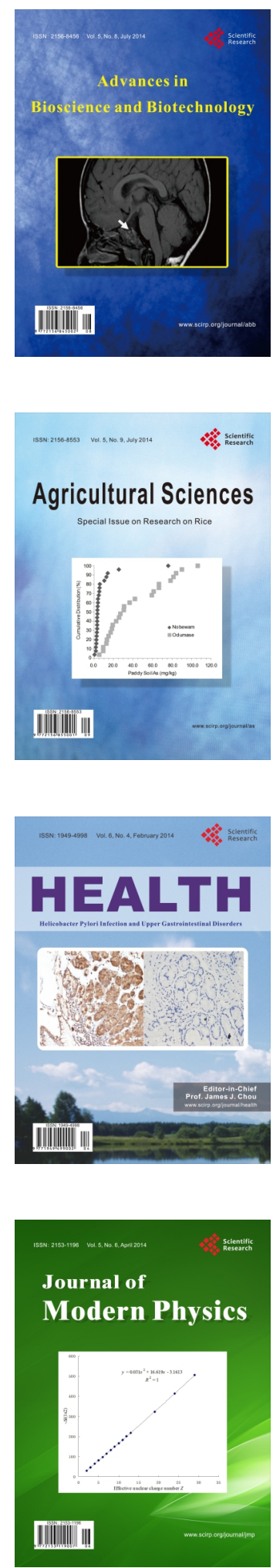
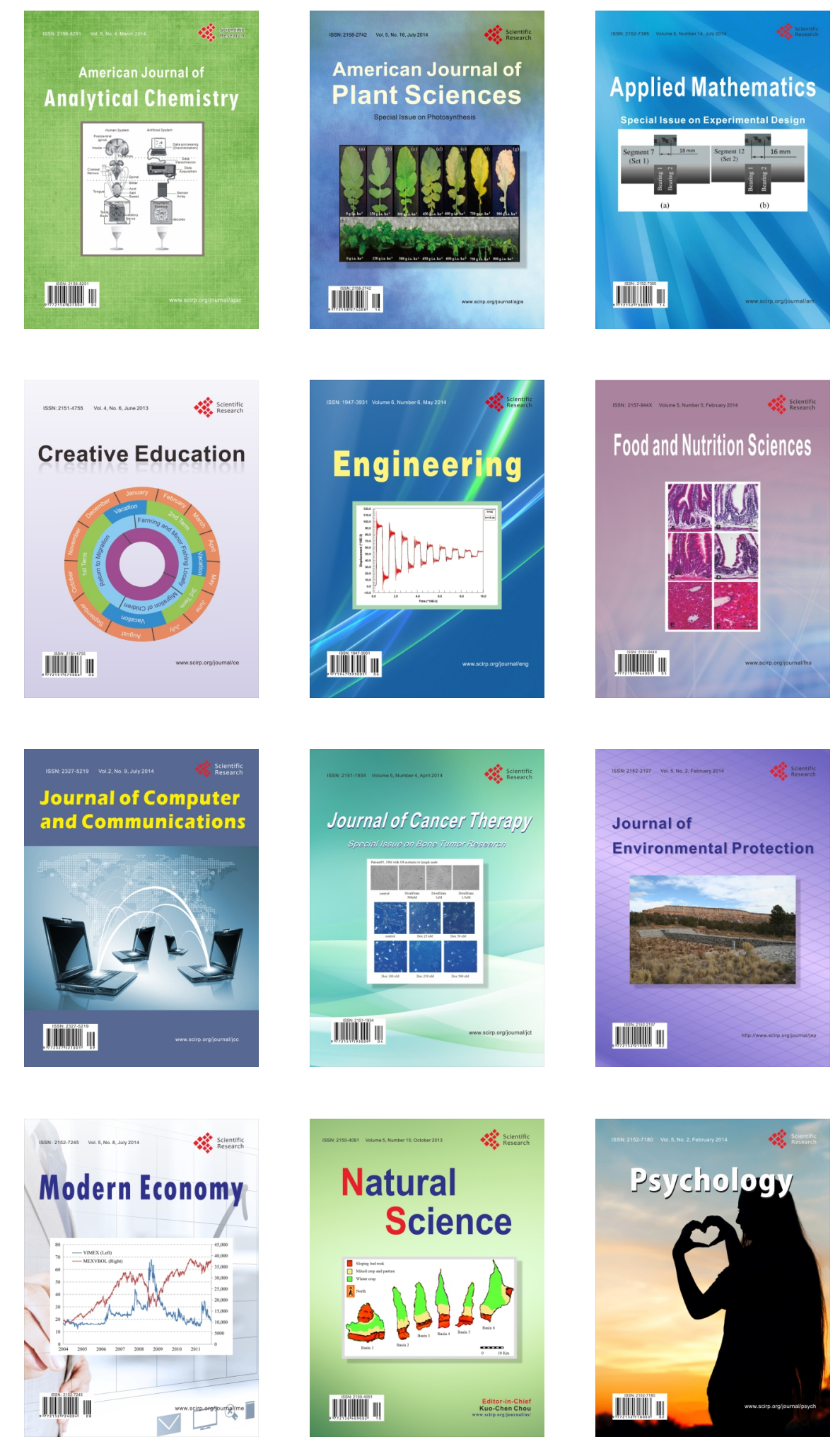